\title{
Optimization of Fractional Frequency Reuse in Long Term Evolution Networks
}

\author{
Dimitrios Bilios $^{1,2}$, Christos Bouras ${ }^{1,2}$, Vasileios Kokkinos ${ }^{1,2}$, Andreas Papazois ${ }^{1,2}$, Georgia Tseliou ${ }^{1,2}$ \\ ${ }^{1}$ Computer Technology Institute \& Press "Diophantus", Patras, Greece \\ ${ }^{2}$ Computer Engineering \& Informatics Dept., Univ. of Patras, Greece \\ bilios@ceid.upatras.gr, bouras@cti.gr, kokkinos@cti.gr, papazois@ceid.upatras.gr, tseliou@cti.gr
}

\begin{abstract}
In cellular systems, Fractional Frequency Reuse (FFR) partitions each cell into two regions; inner region and outer region and allocates different frequency band to each region. Since the users at the inner region are less exposed to inter-cell interference, the frequency resources in each inner region can be universally used. Based on this frequency band allocation, FFR may reduce channel interference and offer large system capacity. This paper proposes a mechanism that selects the optimal FFR scheme based on the user throughput and user satisfaction. In detail, the mechanism selects the optimal size of the inner and outer region for each cell as well as the optimal frequency allocation between these regions that either maximizes the mean user throughput or the user satisfaction. The mechanism is evaluated through several simulation scenarios.
\end{abstract}

Keywords-long term evolution; fractional frequency reuse; orthogonal frequency division multiple access;

\section{INTRODUCTION}

Orthogonal Frequency Division Multiple Access (OFDMA) has become an attractive technology in recent years and it is part of various system standards for mobile communications. This happens because each terminal occupies a subset of subcarriers (called OFDMA traffic channel) and each traffic channel is assigned exclusively to one user at any time [1].

Several techniques with different degrees of complexity can be considered for out-of-cell interference mitigation in OFDMA systems. OFDMA provides a degree of freedom by allowing dynamic assignment of channels/subcarriers to different users at different time instances, to take advantage of the channel response variations among different users on different channels [1]. Sub-channelization implies that a significant fraction of the power is used on only a portion of the bandwidth used to serve the weak user even though universal reuse. Nevertheless, neighboring sectors should assign orthogonal subcarriers to cell edge users and it is important to consider interference when assigning subcarriers to users.

One of the key characteristics of a cellular network is the ability to reuse frequencies in order to increase both capacity and coverage. Fractional Frequency Reuse (FFR) is discussed in the OFDMA-based network, such as the Long Term Evolution (LTE), to overcome the Co-Channel Interference (CCI) problems [2]. In FFR the cell space is divided into two regions: inner, which is close to the Base Station (BS) and outer, which is situated to the borders of the cell. The whole frequency band is divided into several sub-bands, and each subband is differently assigned to inner and outer region of the cell respectively. As a result of FFR, intra-cell interference is eliminated, and inter-cell interference is substantially reduced [3]. At the same time the system throughput is enhanced. Various reuse factors and interference mitigation levels can be achieved by adjusting either the bandwidth proportion assigned to each region or the transmission power of each band.

It should be noted that the majority of the related work regarding FFR in the context of OFDMA systems has mainly been discussed in cellular network standardization for 3GPP and 3GPP2 [4]. This idea was first proposed for GSM networks, which is analyzed by the authors of [5] and has been adopted in the WiMAX forum [2]. FFR is also proposed in the course of the LTE standardization. In the research works [6] and [7], the focus lies on practically implementable algorithms. The authors use the full set of available resources in the reuse 1 areas and one third of the same resources in the reuse 3 areas. In [6] the reuse 1 areas are covered with a reduced power level, while in [7] the transmit power of interfering base stations is reduced. Finally in [8] a novel FFR scheme for multi-cell OFDMA systems that improves channel capacity is proposed.

Main goal of this paper is to propose and evaluate an interference management FFR mechanism for OFDMA macrocell networks. The mechanism calculates the optimal FFR scheme based on two parameters: user throughput and user satisfaction. The proposed mechanism successively checks the inner cell radius and the inner cell frequency and calculates the per-user Signal to Interference plus Noise Ratio (SINR), capacity and throughput. These values are then used in order to calculate the cell mean throughput and the user satisfaction. Finally, the mechanism selects the optimal FFR scheme that either maximizes the cell mean throughput or the user satisfaction. The paper also presents several simulation scenarios in order to evaluate the proposed FFR mechanism.

The rest of this paper is organized as follows. Basic theoretical background regarding FFR is explained in Section II. Section III describes the procedure used for the calculation of SINR, throughput and user satisfaction and introduces the proposed mechanism. The evaluation of the mechanism and the simulation results are described in Section IV, while the conclusions and ideas for future work are shown in Section V. 


\section{FRACTIONAL FREQUENCY REUSE}

In FFR, in order to ensure that the mutual interference between users and base stations remains below a harmful level, adjacent cells use different frequencies. In fact, a set of different frequencies is used for each cluster of adjacent cells. Cluster patterns and the corresponding frequencies are reused in a regular pattern over the entire service area. The closest distance between the centers of two cells using the same frequency (in different clusters) is determined by the choice of the cluster size and the lay-out of the cell cluster. This distance is called the frequency reuse distance.

One of the main objectives of LTE is to achieve high spectral efficiency, meaning the use of the whole of the system's bandwidth in all cells. This approach is called Frequency Reuse 1, and it is considered the simplest scheme; all sub-bands of the available bandwidth are allocated to each cell. In Frequency Reuse 3, the system bandwidth is divided into 3 equal sub-bands; each one of these is allocated to cells in a manner that no other surrounding cell is using the same subband. Full frequency reuse in each cell can exempt the necessity of advance frequency planning among different cells, and the frequency reuse patterns can be dynamically adapted on a frame-by-frame basis in each cell. In this work we study a sub-case of these approaches, which we analyze below.

Firstly we define an LTE OFDMA multi-cellular network. Our main objective is to apply FFR in order to improve the SINR and throughput and simultaneously reduce CCI. An indicative architecture and frequency band allocation are depicted in Figure 1.

In detail, each macrocell of the architecture is divided into two regions; inner and outer region. The total available bandwidth of the system is split into four uneven spectrums denoted by A (yellow), B (red), C (green) and D (blue). Spectrums B, C and D have equal bandwidth and are allocated in outer regions with Frequency Reuse 3. On the other hand, spectrum $\mathrm{A}$ is allocated in all inner regions with Frequency Reuse 1 (Figure 1). The frequency resources in all inner regions are universally used, since the inner region users are less exposed to inter-cell interference. This frequency allocation can eliminate the intra-cell interference and greatly reduce the inter-cell interference. The users are assumed to be distributed uniformly in the cells of the topology.

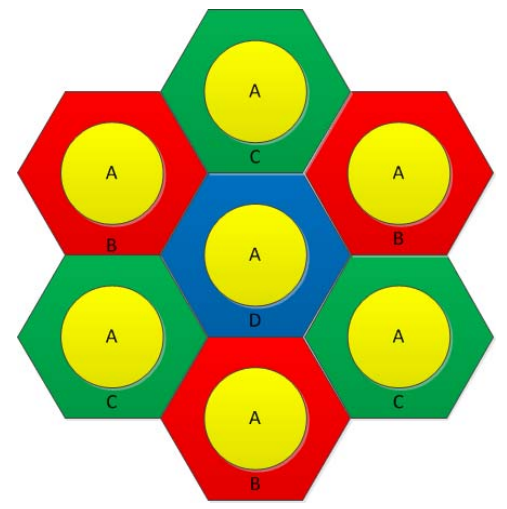

Figure 1. Proposed frequency band allocation.

\section{SYSTEM MODEL AND MECHANISM DESCRIPTION}

\section{A. Throughput and User Satisfaction Calculation}

In this section we describe the theoretical approach to calculate the SINR, throughput and user satisfaction factor.

We assume that the overall network is composed of $N$ adjacent cells. Each cell contains a number of users seeking to share a group of subcarriers. We distinguish the case where a user is found in the inner region of the cell or in the outer. In a typical OFDMA cellular network, for a user $x$ who is served by a base station $b$ on subcarrier $n$, the related SINR is given by the following equation [9]:

$$
\operatorname{SINRx,n=\frac {G_{b,x}P_{b,n}h_{b,x,n}}{\sigma _{n}{}^{2}+\sum _{j}^{k}G_{j,x}P_{j,n}h_{j,x,n}}}
$$

In (1), $G_{b, x}$ refers to the path loss associated with the channel between user $x$ and base station $b, P_{b, n}$ is the transmit power of the base station on subcarrier $n, h_{b, x, n}$ is the exponentially distributed channel fast fading power and $\sigma_{n}{ }^{2}$ is the noise power of an Additive White Gaussian Noise (AWGN) channel. Symbols $k$ and $j$ refer to the set of all the interfering base stations (i.e. base stations that are using the same sub-band as user $x$ ). Their physical meaning is that $j$ is the cell index and $k$ the number of co-channel cells. In our analysis, we assume that equal transmit power is applied, $P_{b, n}=P$ for all base stations. The coefficient $h_{b, x, n}$ is replaced by its mean value $\left(h_{b, x, n}=1\right)$ in equation (1).

The interference that occurs comes from disjoint sets of downlinks in the inner and outer region. A transmission in a cell inner region that is assigned a specific frequency band causes interference only to inner users of other cells that are assigned the same band. Furthermore, it is necessary to distinguish two categories of base stations. The first consists of all interfering base stations transmitting to cell inner users on the same sub-band as user $x$ and the second consists of all interfering base stations transmitting to cell-edge users on the same sub-band as user $x$.

After the SINR estimation, we proceed with the throughput calculation. The capacity of user $x$ on subcarrier $n$ can be calculated by the following equation [8]:

$$
C_{x, n}=\Delta f \cdot \log _{2}\left(1+\operatorname{SINR}_{x, n}\right)
$$

where, $\Delta f$ refers to the available bandwidth for each subcarrier divided by the number of users that share the specific subcarrier. Moreover, the throughput of the user $x$ can be expressed as follows:

$$
T_{x}=\sum_{n} \beta_{x, n} C_{x, n}
$$


where, $\beta_{x, n}$ represents the subcarrier assigned to macro user $x$. When $\beta_{x, n}=1$, the subcarrier $n$ is assigned to macro user $x$. Otherwise, $\beta_{x, n}=0$.

Moreover, in order to evaluate the performance of our experiments we define the term User Satisfaction (US) as the sum of the users' throughput divided by the product of the maximum user's throughput and the number of users $(X)$. This metric physically presents how close the user's throughput is to the maximum throughput in the area. Specifically:

$$
U S=\frac{\sum_{x=1}^{X} T_{x}}{\text { max_user_throughput }{ }^{*} X}
$$

US ranges between 0 and 1 . When US approaches 1 , all users in the corresponding cell experience similar throughput, while when US approaches 0 , there is a big difference in the throughput achieved by the users in the cell.

\section{B. Mechanism Description}

The mechanism assumes a number of multicast users that are uniformly distributed in the topology. In order to find the optimal FFR scheme, the mechanism divides each cell into two regions and calculates the total throughput and US for the following 26 Frequency Allocations (FA), assuming Frequency Reuse 1 and 3 for the inner and the outer region respectively:

- FA1: All (25) subcarriers are allocated in inner region. No subcarriers are allocated in outer region.

- FA2: 24 subcarriers are allocated in inner region. $1 / 3$ subcarrier allocated in outer region.

- $\quad \cdots$

- FA25: 1 subcarrier allocated in inner region. 24/3 subcarrier allocated in outer region.

- FA26: No subcarriers allocated in inner region. $25 / 3$ subcarriers allocated in outer region.

For each FA, the mechanism calculates the per-user throughput and the mean throughput and US. This procedure is repeated for successive inner cell radius ( 0 to $R$, where $R$ is the cell radius). Finally, the mechanism selects the optimal FFR schemes that maximize the mean throughput and US.

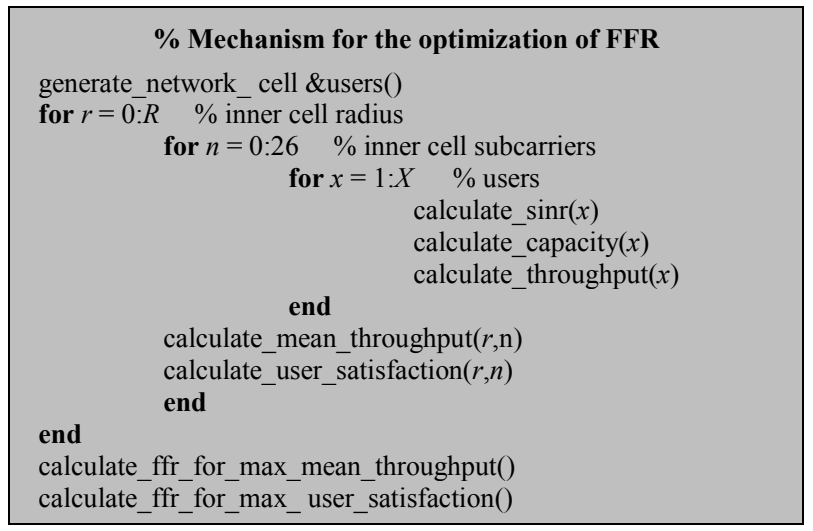

The pseudo-code of the proposed FFR mechanism is presented above. The complexity and the running time of the algorithm are proportional to the number of users multiplied by the number of cells in the topology. This means that that the complexity can be expressed as $\mathrm{O}(\#$ users *\# cells $)$.

\section{PERFORMANCE STUDY}

\section{A. Simulation Parameters}

The simulation parameters that are necessary for the conduction of the experiment are presented in Table I. In detail, we consider a system with $10 \mathrm{MHz}$ of bandwidth (i.e. LTE) divided into 25 sub-carriers each having a bandwidth of 375 $\mathrm{KHz}$. The scenario assumed is urban canyon macro, which exists in dense urban areas served by macro-cells. Path losses are calculated according to Cost-Hata Model [10] and the correlation distance of the shadowing is set to $40 \mathrm{~m}$ [8].

TABLE I. SIMULATION PARAMETERS

\begin{tabular}{lll}
\hline \hline Parameter & Units & Value \\
\hline System bandwidth & $\mathrm{MHz}$ & 10 \\
Subcarriers & & 25 \\
Subcarriers' bandwidth & $\mathrm{KHz}$ & 375 \\
Carrier frequency & $\mathrm{MHz}$ & 2000 \\
Cell Radius & $\mathrm{m}$ & 250 \\
Correlation distance & $\mathrm{m}$ & 40 \\
Channel model & & $3 \mathrm{GPP}$ Typical Urban \\
Path loss & $\mathrm{dB}$ & Cost 231 Hata Model \\
BS transmit power & $\mathrm{dBm}$ & 46 \\
Power Noise Density & $\mathrm{dbm} / \mathrm{Hz}$ & -174 \\
\hline \hline
\end{tabular}

\section{B. Optimal FFR based on Cell Mean Throughput}

The first simulation experiment presents the operation of the mechanism when selecting the FFR scheme that maximizes the cell mean throughput. The scenario assumes that the users are distributed uniformly in the topology, which consists of 16 cells (Figure 2). We will focus on one cell of the topology, (second row and third column) which is highlighted in Figure 2. The specific cell contains 30 users. As Figure 2 presents, the FFR scheme that maximizes the mean throughput for the examined cell consists of two regions, the inner region (yellow) has $93.5 \mathrm{~m}$ radius and contains 3 users while the rest cell area (red) constitutes the outer region and contains 27 users.

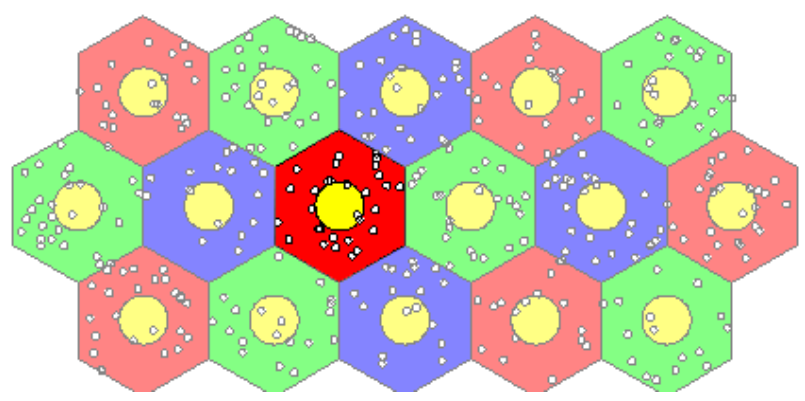

Figure 2. Deployment of optimal FFR scheme based on cell mean throughput (optimal inner cell radius $=93.5 \mathrm{~m}$ ). 


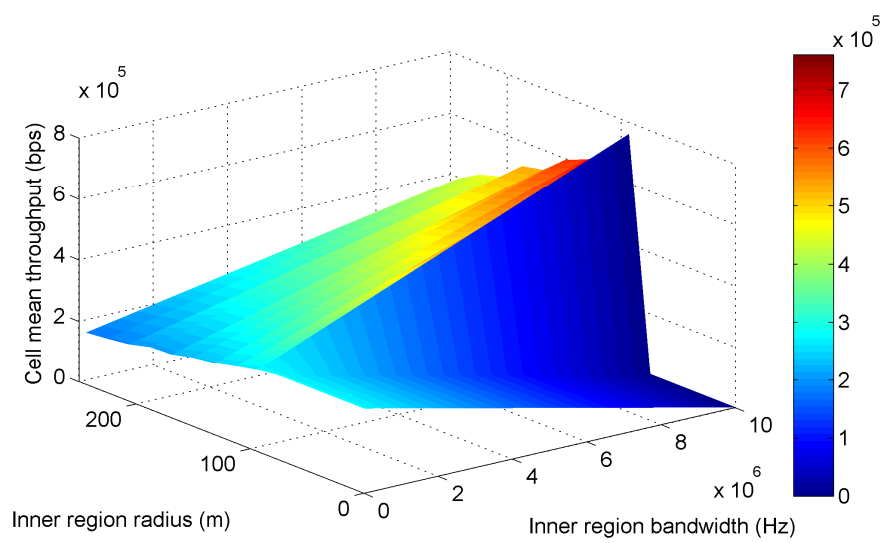

Figure 3. 3D representation of cell mean throughput vs. inner region bandwidth vs. inner region radius.

Figure 3 depicts the 3D representation of the cell mean throughput against the inner region bandwidth and radius. According to this figure, the highest values of mean throughput are observed for inner region radius between $80 \mathrm{~m}$ and $150 \mathrm{~m}$ and for inner region bandwidth between $6 \mathrm{MHz}$ and $10 \mathrm{MHz}$. However, as mentioned the FFR scheme that maximizes the cell mean throughput occurs for inner region radius equal to $93.5 \mathrm{~m}$ and inner region bandwidth equal to $10 \mathrm{MHz}$.

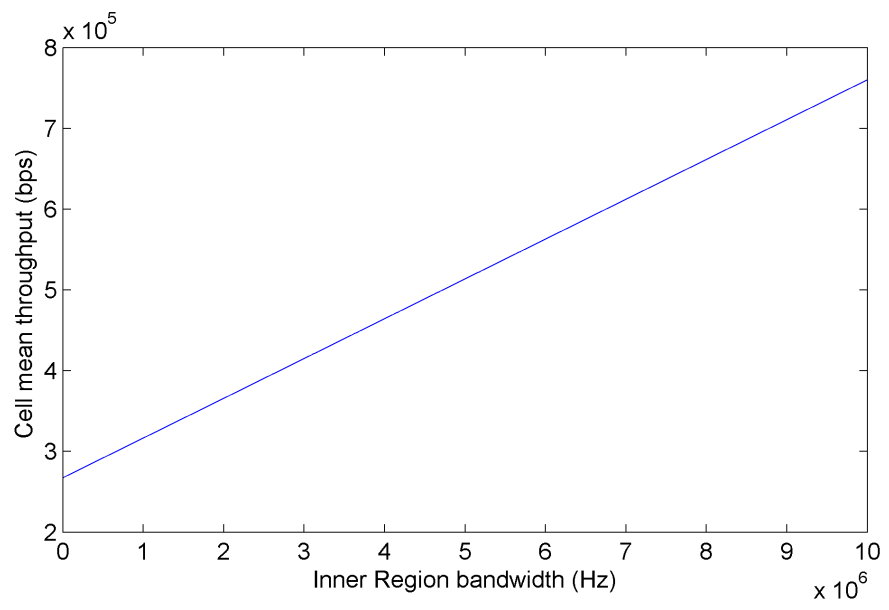

Figure 4. Cell mean throughput vs. inner region bandwidth for the optimal inner region radius.

Figure 4 presents the cell mean throughput against the inner region bandwidth for the optimal inner cell radius. We observe that the mean throughput increases linearly as the bandwidth allocated in the inner region increases. In addition, the maximum mean throughput occurs when all the available bandwidth is allocated to the three users of the inner region. On the other hand, the mechanism does not allocate bandwidth to the remaining 27 users of the outer region.

\section{Optimal FFR based on User Satisfaction}

In order to overcome the unfairness in bandwidth allocation among the users in different regions of the cell, in Section III.A we introduced the user satisfaction (US) metric, which ensures that all users in a cell will experience similar values of throughput. The experiment that follows presents the operation of the mechanism when selecting the FFR scheme that maximizes the US. The topology and user distribution are in accordance to the previous experiment. However, as depicted in Figure 5 the optimal FFR scheme occurs when the inner region has $150 \mathrm{~m}$ radius and contains 13 users.

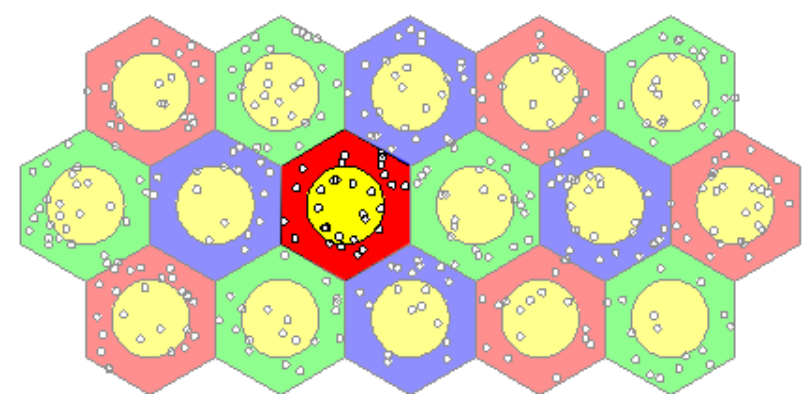

Figure 5. Deployment of optimal FFR scheme based on user satisfaction (optimal inner region radius $=150 \mathrm{~m}$ ).

Figure 6 presents the US as a function of the inner region bandwidth and the inner region radius. We have to remind that a value of US metric closer to 1 is the optimal since it leads to small differences in the values of users' throughput. Therefore, according to Figure 6 the optimal FFR scheme corresponds to the deployment with $150 \mathrm{~m}$ inner region radius and $2.06 \mathrm{MHz}$ inner region bandwidth that results in US equal to 0.756 .

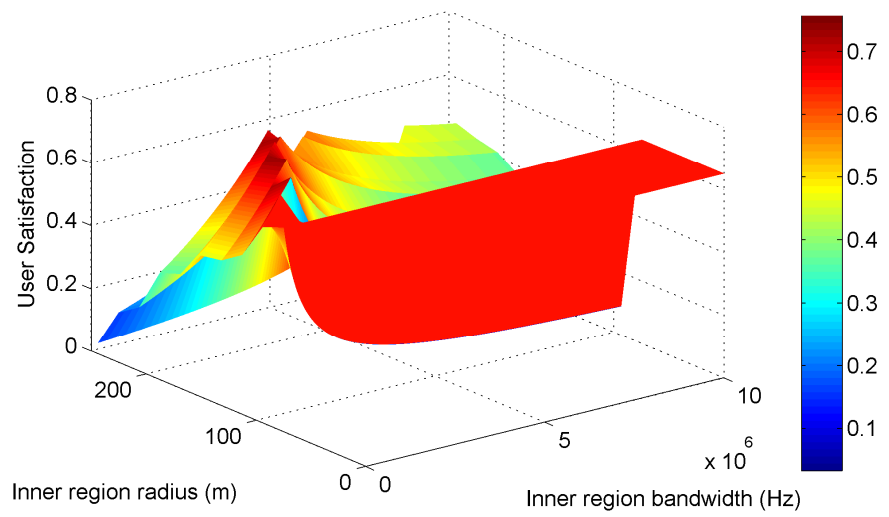

Figure 6. 3D representation of user satisfaction vs. inner region bandwidth vs. inner region radius.

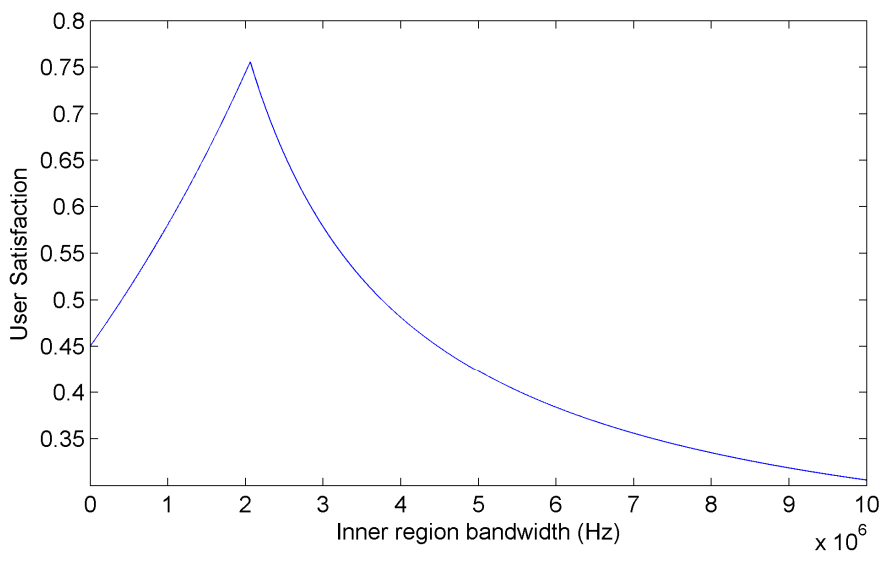

Figure 7. User satisfaction vs. inner region bandwidth for the optimal inner region radius. 
Finally, Figure 7 presents the way that the user satisfaction changes as the inner region bandwidth increases. The inner region radius equals to $150 \mathrm{~m}$. According to this figure, as the bandwidth allocated to the inner region increases from 0 to $2.06 \mathrm{MHz}$ the US increases and reaches the maximum value (0.756) for bandwidth equal to $2.06 \mathrm{MHz}$. Higher values of inner region bandwidth lead to US decrement, while allocating all the available bandwidth to the inner region leads to the minimum value of US.

\section{Comparison of the Approaches}

This section makes a direct comparison between the FFR schemes that presented in sections IVB and IVC. More specifically, Figure 8 presents the per-user throughput achieved by each approach.

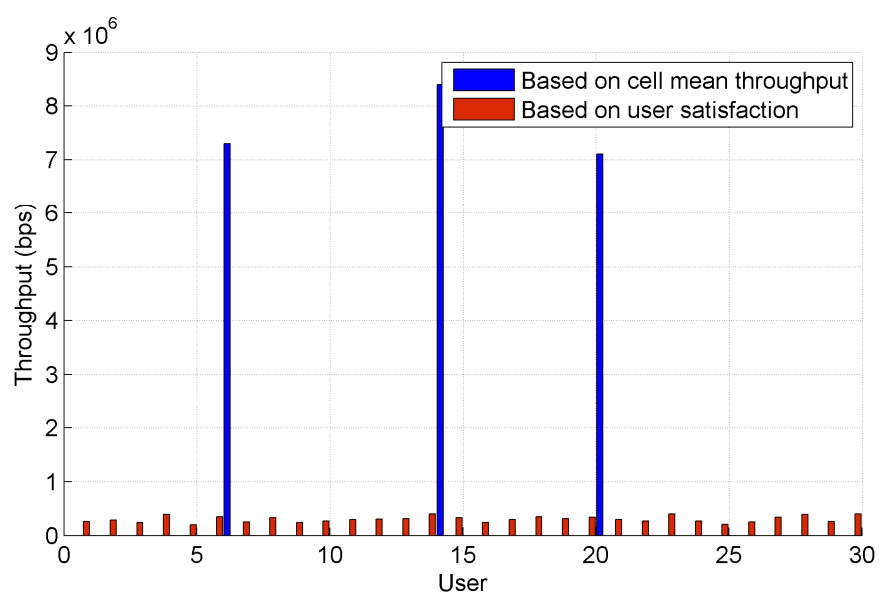

Figure 8. Per-user throughput for the two approaches (based in cell mean throughput and user satisfaction).

According to Figure 8, the optimal FFR scheme that is based on the maximization of cell mean throughput allocates the available bandwidth to the three users that are located in the inner region and serves only these users with very high throughput. However, this approach does not guarantee that all the users will be served. Indeed, the 27 users that are located in the outer region have throughput equal to 0 . On the other hand, the optimal FFR scheme that is based on the maximization of user satisfaction ensures that all users will share similar values of throughput irrespectively of their location in the cell. Indeed, even if the mean throughput is lower compared to the first approach, we can conclude that the FFR scheme that is based on the maximization of user satisfaction presents a better and fairer overall behavior.

\section{CONCLUSIONS \& FUTURE WORK}

In this paper we proposed an interference management FFR mechanism that calculates the per-user SINR, capacity and throughput and uses these values to calculate the cell mean throughput and user satisfaction. Based on these values the mechanism may follow two different approaches for selecting the optimal FFR scheme. The first approach is based on maximization of the cell mean throughput and the second is based on the maximization of user satisfaction. Moreover, we have conducted simulation experiments in order to examine the operation of the mechanism and compare the two approaches. As a general conclusion, we can say that the approach that is based on the maximization of user satisfaction presents a better and fairer overall behavior.

The step that follows this work could be the extension of the mechanism in order to support the users' mobility. The extended mechanism could also monitor the network and users conditions and use them as input to decide the appropriate frequency allocation between the inner and outer region of the cell.

\section{REFERENCES}

[1] IEEE standard for local and metropolitan area networks, "Part 16: Air interface for fixed and mobile broadband wireless access systems", 28 February 2006.

[2] Mobile WiMAX-Part II: A comparative analysis, Tech. Rep., WiMAX Forum, May 2006.

[3] M.C. Necker, "Local Interference Coordination in Cellular OFDMA Networks" IEEE 66th Vehicular Technology Conference, 2007. VTC2007 Fall. 2007.

[4] 3GPP, Radio access network work group1 contributions, http://www.3gpp.org, September 2005.

[5] K. Begain, G. I. Rozsa, A. Pfening, and M. Telek, Performance analysis of GSM networks with intelligent underlay-overlay, in Proc. 7th International Symposium on Computers and Communications (ISCC 2002), Taormina, Italy, 2002, pp. 135141.

[6] 3GPP TSG RAN WG1\#42 R1-050841, Further analysis of soft frequency reuse scheme, Huawei, Tech. Rep., 2005.

[7] 3GPP TSG RAN WG1\#42 R1-050764, Inter-cell interference handling for E-UTRA, Ericsson, Tech. Rep., September 2005.

[8] H. Lei, L. Zhang, X. Zhang, and D. Yang, "A Novel Multi-Cell Ofdma System Structure Using Fractional Frequency Reuse", In proceedings of the 18th Annual IEEE International Symposium on Personal, Indoor and Mobile Radio Communications (PIMRC'07).

[9] 3GPP TSG-RAN, "3GPP TR 25.814, Physical Layer Aspects for Evolved UTRA (Release 7)", v1.3.1 (2006-05).

[10] S. Saunders, Antennas and Propagation for Wireless Communication Systems, Wiley, 2000, 409 p. 